'Departamento de Nefrología. Escuela de Medicina, Facultad de Medicina. Pontificia Universidad Católica de Chile. Santiago, Chile. ${ }^{2}$ Departamento de Medicina Interna. Escuela de Medicina, Facultad de Medicina. Pontificia Universidad Católica de Chile. Santiago, Chile.

${ }^{3}$ Sección de Nefrología. Hospital Naval Almirante Nef. Viña del Mar, Chile.

Trabajo no recibió financiamiento. Los autores declaran no tener conflictos de interés.

Recibido el 8 de enero de 2020 aceptado el 4 de junio de 2020 .

Correspondencia a: Dr. Juan Pablo Huidobro Diagonal Paraguay 362 4to piso. Santiago, Chile. jphuidob@uc.cl

\section{Nueva fórmula para estimar creatininuria en población chilena}

\author{
JUAN PABLO HUIDOBRO ESPINOSA ${ }^{1,3}$, \\ RODRIGO ANDRÉS SEPÚLVEDA ${ }^{1}$, GONZALO MAURICIO MUÑOZ ${ }^{2}$
}

\section{A new equation to predict urine creatinine values using data from Chilean population}

Background: Evaluation of 24-hour collection accuracy is based on urinary creatinine excretion (UCr), usually using wide ranges indexed by weight. Equations that predict the expected UCr are also available. Aim: To generate an equation for estimating UCr in Chilean population and evaluate its performance in comparison to existing formulas. Material and Methods: A total of 464 24-hour urine collections from outpatients aged between 15 and 88 years old were used. Ninety percent of collections $(n=418)$ were randomly extracted to assess the association between absolute UCr values with sex, age, height and weight of participants. A formula was created to estimate the 24-hour UCr using a multiple linear regression model. In the remaining $10 \%$ of urine collections $(n=46)$, the performance of this formula and others reported in the literature were tested. Results: Age, sex and weight were significantly associated with 24-hour UCr values. The new equation was able to predict $U C r$ values with a similar accuracy than CKD-EPI and Walser equations and outperformed other equations. Conclusions: Our equation developed with Chilean values predicts 24-hour UCr values accurately.

(Rev Med Chile 2020; 148: 734-739)

Key words: Creatinine; Glomerular Filtration Rate; Kidney Function Tests; Renal Elimination.

\section{L} as recolecciones de orina de $24 \mathrm{~h}$ son una herramienta ampliamente utilizada en nefrología desde comienzos del siglo $\mathrm{XX}^{1}$, utilizándose para estimar la tasa de filtración glomerular, cuantificar proteinuria, estudiar funciones tubulares, realizar estudio de litiasis urinaria y evaluar adherencia a recomendaciones nutricionales. No obstante, el principal reparo para esta herramienta es la necesidad de lograr una adecuada recolección, con series que describen recolecciones óptimas incluso en menos de 50\% de los casos ${ }^{2}$.

La evaluación de la validez de la recolección se realiza habitualmente a partir de los valores de creatininuria (UCr), utilizándose frecuentemente rangos amplios y normalizados por peso, que subestiman errores en la recolección. Existen, además de los rangos de referencia ajustados por peso, fórmulas que predicen la UCr esperada y que incorporan edad, sexo y datos antropométricos. Hasta la fecha, sin embargo, no hay reportes de los valores normales de UCr en población chilena (ni latina) ni tampoco una fórmula para estimarla.

El objetivo primario de este trabajo fue desarrollar una fórmula que permita predecir los valores de UCr de 24 h en población chilena. El objetivo secundario fue comparar la capacidad de predicción de la UCr de 24 h de la nueva fórmula propuesta con las 8 fórmulas actualmente disponibles en la literatura. 


\section{Pacientes y Métodos}

Se incluyeron todas las recolecciones de orina de 24 h realizadas entre enero de 2015 y junio de 2019 en el Laboratorio Clínico de la Red de Salud UC-Christus. Se registraron la edad, sexo, lugar de recolección (ambulatorio u hospitalizado), volumen urinario, peso y talla de los pacientes. En todas las muestras de orina se midió creatinina.

Para el desarrollo y validación de la fórmula se excluyeron aquellas recolecciones de pacientes menores de 15 años y las que no cumplían con los rangos esperados de UCr ajustados por peso establecidos por el laboratorio de la Clínica Mayo: $13-29 \mathrm{mg} / \mathrm{kg} / 24 \mathrm{~h}$ para hombres y $9-26 \mathrm{mg} / \mathrm{kg} / 24 \mathrm{~h}$ para mujeres ${ }^{3}$. También, se excluyeron las recolecciones realizadas en pacientes hospitalizados y aquellos que tuvieran más de una recolección en un plazo menor de 12 meses.

Para la confección del modelo predictivo se buscó que los coeficientes $\beta$ de las variables predictoras tuvieran una elevada precisión (intervalos de confianza $95 \%$ estrechos). Considerando un error alfa de $5 \%$, una potencia estadística de $95 \%, 6$ predictores en la fórmula y un tamaño del efecto pequeño para el coeficiente de determinación $(0,05)$; se estimó un tamaño muestral para la regresión lineal múltiple de 408 . De las múltiples formas existentes para validar modelos de regresión ${ }^{4}$, actualmente se recomienda utilizar métodos de división de datos ${ }^{5}$. Por lo tanto, del total de la población estudiada, se extrajo aleatoriamente $90 \%(\mathrm{n}=418)$ para la creación de la ecuación (población de formulación) y el $10 \%(\mathrm{n}=46)$ restante se utilizó como muestra para validación.

En la población de formulación se evaluó la asociación de los valores absolutos de UCr de $24 \mathrm{~h}$ con: sexo, edad, talla y peso de los pacientes y se desarrolló una fórmula para estimar la $\mathrm{UCr}$ de $24 \mathrm{~h}$ mediante un modelo de regresión lineal múltiple con selección backward de las variables.

La capacidad de predicción de la UCr de $24 \mathrm{~h}$ con la fórmula propuesta y las 8 existentes se probó en $10 \%$ de los pacientes de la base de datos no utilizados para la construcción de la nueva fórmula.

Para el análisis univariado de variables cuantitativas se emplearon promedios con su respectiva desviación estándar o rango, y frecuencias en el caso de variables cualitativas. El análisis bivariado se realizó mediante correlación lineal de Pearson o t de student según el caso. Para el análisis multivariado se empleó regresión lineal múltiple.

El rendimiento de las fórmulas se realizó mediante análisis de asociación, a través de correlación lineal de Pearson y análisis de concordancia, para lo cual se utilizaron el coeficiente de correlación de Lin y gráficos de Bland-Altman. Se consideró estadísticamente significativo un valor $\mathrm{p}<0,05$.

El análisis estadístico se realizó con el software Stata V.14.

Este estudio cuenta con la aprobación del comité de ética-clínico de la Facultad de Medicina de la Pontificia Universidad Católica de Chile.

\section{Resultados}

Se analizaron 692 recolecciones de orina realizadas en el período de estudio. Luego de aplicados los criterios de exclusión se obtuvieron 464 recolecciones de orina, de las cuales se seleccionó aleatoriamente el 90\% $(\mathrm{n}=418)$ para análisis estadísticos y creación de la fórmula, y sobre el $10 \%$ restante $(\mathrm{n}=46)$ se realizaron pruebas de rendimiento y validación interna.

La población analizada incluyó pacientes entre 15 y 88 años (media y desviación estándar (DS) 51,8 y 17,2 , respectivamente, con $63 \%$ de mujeres y una media de creatinina de $1 \mathrm{mg} / \mathrm{dL}$ (DS 0,46, rango 0,33-4,99) (Tabla 1).

La media de UCr de $24 \mathrm{~h}$ fue de $1.590 \mathrm{mg}(19,9$ $\mathrm{mg} / \mathrm{kg})$ con un rango entre 840-2.900 $\mathrm{mg}(13,7-$ $28,7 \mathrm{mg} / \mathrm{kg})$ para hombres y $1.029 \mathrm{mg}(15,97 \mathrm{mg} /$ $\mathrm{kg}$ ), con rango entre $370-1.710 \mathrm{mg}(9-25,5 \mathrm{mg} /$ $\mathrm{kg}$ ) para mujeres. $\mathrm{La} \mathrm{UCr}$ de $24 \mathrm{~h}$ promedio de las mujeres fue significativamente menor que la de los hombres $(\mathrm{p}<0,01)$. La edad presentó correlación lineal inversa con los valores de UCr de

\section{Tabla 1. Características de los 418 pacientes utilizados para elaboración de la fórmula}

\begin{tabular}{|lc|}
\hline Edad (años) (media \pm DS, rango) & $51,8 \pm 17,2(15-88)$ \\
\hline Sexo femenino, $\mathrm{n}(\%)$ & $264(63,2 \%)$ \\
\hline Peso $(\mathrm{kg})$ (media $\pm \mathrm{DS}$, rango) & $70,8 \pm 14,5(28-130)$ \\
\hline Talla $(\mathrm{cm})$ (media $\pm \mathrm{DS}$, rango) & $164 \pm 9,7(134-194)$ \\
$\begin{array}{l}\text { Creatinina plasmática }(\mathrm{mg} / \mathrm{dL}) \\
\text { (media } \pm \mathrm{DS}, \text { rango) }\end{array}$ & $1,00 \pm 0,46$ \\
\hline
\end{tabular}


$24 \mathrm{~h}(\mathrm{r}=-0,23 ; \mathrm{p}=<0,01)$ mientras que el peso $\mathrm{y}$ talla tuvieron correlación lineal positiva $(r=0,67$; $\mathrm{p}<0,01 ; \mathrm{r}=0,62 ; \mathrm{p}<0,01$, respectivamente).

Al realizar una regresión lineal múltiple para la UCr de $24 \mathrm{~h}$; el sexo femenino, edad y peso mantenían las asociaciones anteriormente mencionadas.

El modelo de regresión lineal múltiple desarrollado fue el siguiente:

$\mathrm{UCr} 24 \mathrm{~h}(\mathrm{mg})=345+\left(\right.$ peso $\left.[\mathrm{kg}]^{\star} 12\right)+$ $\left(\right.$ talla $\left.[\mathrm{cm}]^{\star 3}\right)-\left(\right.$ edad $\left.[\text { años }]^{\star 5}\right)-340($ si mujer $)$

Se probó el rendimiento de la fórmula creada (de aquí en adelante "fórmula propuesta") y el de otras reportadas en la literatura (Tanaka ${ }^{6}$, IxCKD-EPI ${ }^{7}$, Cockroft-Gault ${ }^{8}$, Walser ${ }^{9}$, Gerber and Mann $^{10}$, Goldwasser et al. ${ }^{11}$, Rule ${ }^{12}$ y Kawasaki ${ }^{13}$ ), en una población de validación de 46 pacientes ( $56,5 \%$ de mujeres y $43,5 \%$ de hombres), con una media de creatinina de $1,05 \mathrm{mg} / \mathrm{dL}$ (DS 0,43 , rango $0,58-2,47 \mathrm{mg} / \mathrm{dL}$ ).

La UCr de 24 h estimada por la fórmula propuesta tuvo una correlación lineal con la UCr de $24 \mathrm{~h}$ medida de $\mathrm{r}=0,802(\mathrm{p}<0,01)$ (Figura 1). Este valor fue similar a las correlaciones obtenidas por las fórmulas de Ix (grupo CKD-EPI) y Walser; y mejor que el resto de las ecuaciones (Tabla 2). En el análisis de concordancia, todas las fórmulas arrojaron rangos considerables de dispersión (cercanos a $500 \mathrm{mg}$ ), siendo la fórmula propuesta la que posee la menor diferencia promedio (Figura 2 y Tabla 2). Sin considerar las ecuaciones de Rule y Kawasaki, las que no tuvieron correlación lineal estadísticamente significativa con la UCr medida en nuestra población, las fórmulas de Cockroft-Gault y Gerber-Mann son las que tienen mayor diferencia promedio respecto a las mediciones. La fórmula propuesta subestima mínimamente el valor de la creatininuria real, mientras que las fórmulas de Ix, Walser, Goldwasser y Cockroft-Gault la subestiman en mayor medida. La ecuación de Tanaka sobreestima el valor de la creatininuria (Tabla 2).

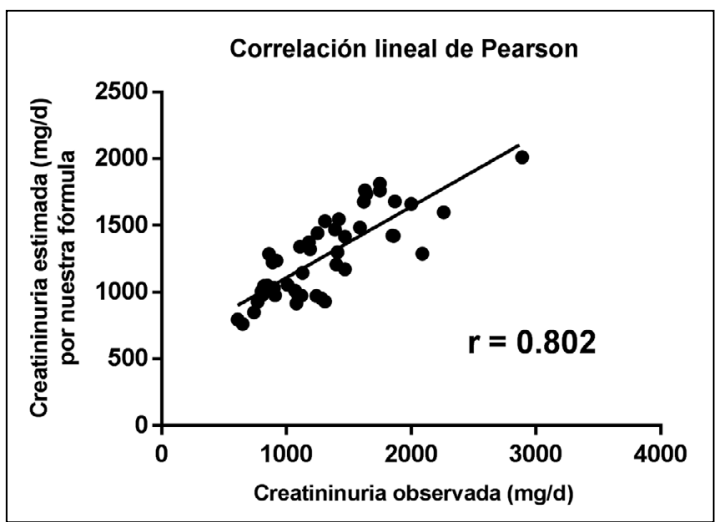

Figura 1. Correlación de nuestra fórmula con la creatininuria medida en población de validación.

Tabla 2. Rendimiento (correlación de Pearson y Lin, P30 y P15) de la fórmula propuesta y las existentes en población chilena

\begin{tabular}{|lccccc|}
\hline Fórmula & $\begin{array}{c}\text { Correlación } \\
\text { lineal } \\
\text { (Pearson) }\end{array}$ & $\begin{array}{c}\text { Coeficiente } \\
\text { correlación } \\
\text { (Lin) }\end{array}$ & $\begin{array}{c}\text { Diferencia promedio } \\
\text { entre creatinina estimada } \\
\text { y medida (mg) }\end{array}$ & P30 & P15 \\
\hline Fórmula propuesta & 0,80 & 0,74 & 36,9 & $86,9 \%$ & $45,6 \%$ \\
\hline Tanaka6 & 0,73 & 0,68 & $-56,9$ & $78,2 \%$ & $39,1 \%$ \\
\hline Ix (CKD-EPI) & 0,80 & 0,74 & 63,9 & $89,1 \%$ & $50 \%$ \\
\hline Cockroft-Gault $^{8}$ & 0,77 & 0,71 & 142 & $78,2 \%$ & $45,6 \%$ \\
\hline Walser $^{9}$ & 0,82 & 0,78 & 53,9 & $91,3 \%$ & $47,8 \%$ \\
\hline Goldwasser & & 0,65 & 60,3 & $76,1 \%$ & $46,5 \%$ \\
\hline Gerber-Mann $^{11}$ & 0,72 & 0,69 & $-196,6$ & $65,2 \%$ & $32,1 \%$ \\
\hline Rule $^{12}$ & 0,78 & $*$ & $*$ & $54,3 \%$ & $23,9 \%$ \\
\hline Kawasaki13 $^{13}$ & $0,22^{*}$ & $*$ & $*$ & $47,8 \%$ & $21,7 \%$ \\
\hline
\end{tabular}

*Dada ausencia de asociación estadísticamente significativa $(p>0,05)$ no se realizaron análisis de concordancia. P30: Porcentaje de las estimaciones con variación menor al 30\% con respecto al valor medido. P15: Porcentaje de las estimaciones con variación menor al 15\% con respecto al valor medido. 

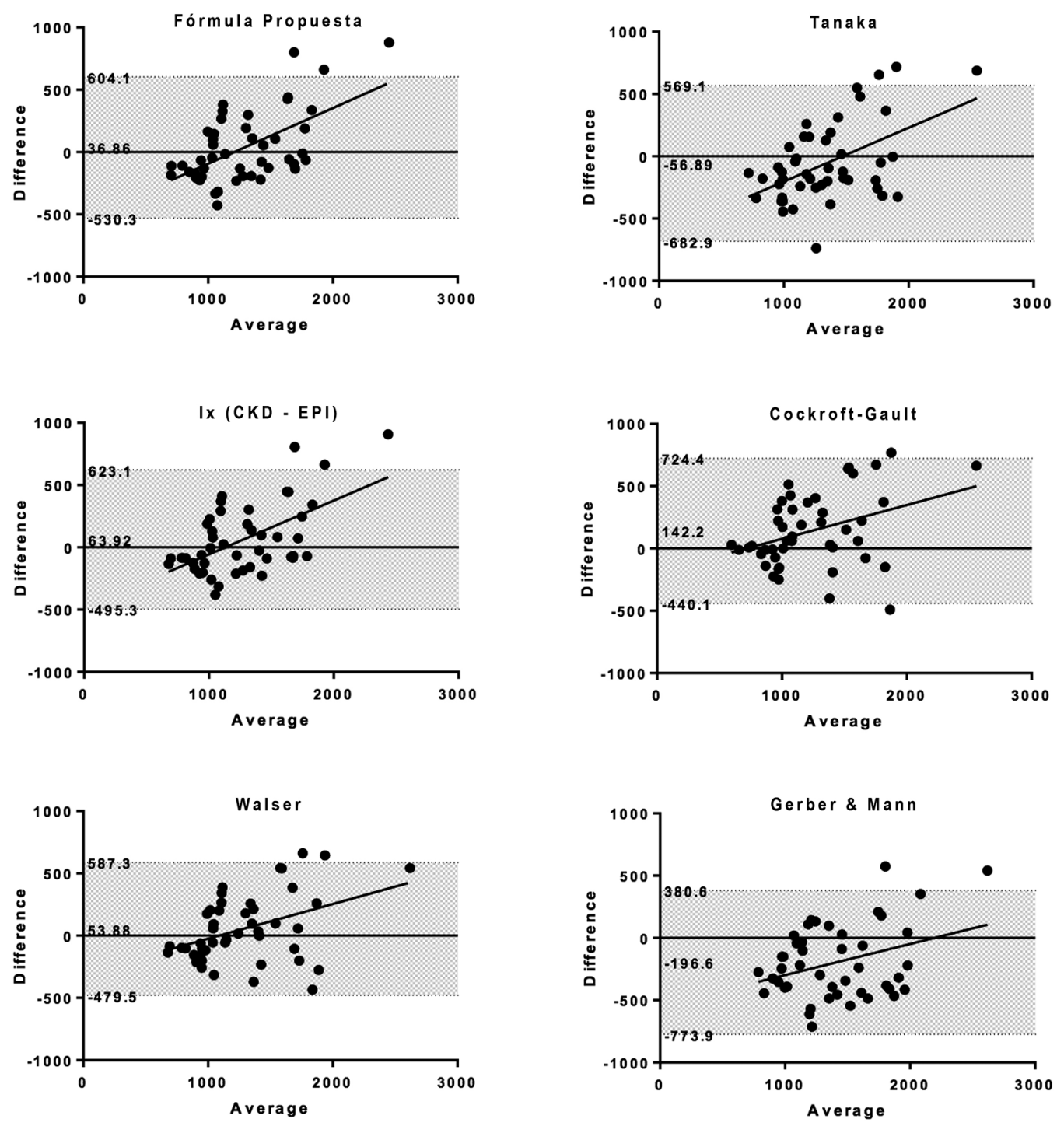

Figura 2. Análisis de concordancia (Bland-Altman) entre creatininuria observada y creatininuria estimada por las diferentes ecuaciones. Se muestra la diferencia entre la creatininuria medida y la observada (eje y) y el promedio entre estos dos parámetros (eje $\mathrm{x}$ ).

Todas las fórmulas subestiman el valor de creatininuria a valores altos de creatininuria medida (Figura 2).

Las fórmulas del grupo CKD-EPI, Walser y la nuestra predijeron valores con menos de $30 \%$ de variación con respecto al valor medido de UCr de $24 \mathrm{~h}$ (P30) en un porcentaje mayor a 85\% de las observaciones. Similar a lo que se ha reportado en la literatura en otras poblaciones ${ }^{14}$, la capacidad de las diferentes ecuaciones para predecir un valor con menos de $15 \%$ de variación con respecto al valor medido de UCr de $24 \mathrm{~h}$ (P15) fue baja, no superando el $50 \%$ de las observaciones para todas las fórmulas (Tabla 2). 


\section{Discusión}

La recolección de orina de $24 \mathrm{~h}$ es ampliamente utilizada en clínica, pero su interpretación está habitualmente limitada por la incertidumbre acerca de la adecuada recolección de orina. Para evaluar la validez de la recolección generalmente se utilizan rangos amplios de $\mathrm{UCr}$ ajustados por peso. Estos se basan en datos existentes desde las décadas de los 60-708,9. En estos estudios, y otros que los sucedieron, se describió que la creatininuria diaria era variable y dependía desde factores nutricionales a emocionales ${ }^{9}$. También, se demostró que a medida que las personas envejecen excretan menos creatinina independiente de su peso $^{8}$ y que las personas de raza negra excretan una mayor cantidad de creatinina diaria que las de origen caucásico ${ }^{10}$.

Además de la interpretación de la idoneidad de la recolección de orina basada en rangos de $\mathrm{UCr}$ ajustados por peso, se han desarrollado hasta la fecha 8 fórmulas para estimar la UCr en poblaciones caucásicas y asiáticas ${ }^{6-13}$, las que también permiten evaluar la validez de una muestra contra valores esperados más precisos de UCr (absolutos o ajustados por peso). Análisis posteriores de las diferentes fórmulas han demostrado que el rendimiento de éstas es en general aceptable, con correlación de Pearson que va entre 0,72 y 0,84 y un rendimiento predictivo con valores estimados con una variación menor a 30\% del valor medido (P30) en general mayores a $75 \%$, dependiente de los criterios de inclusión utilizados para el análisis ${ }^{14}$. También es importante destacar que el rendimiento de las ecuaciones es dependiente de la población a partir de la cual se formuló ${ }^{14}$. Incluso, grupos más específicos de pacientes, como aquellos con glomerulopatías con pérdida de proteínas, podrían excretar cerca del $50 \%$ de la UCr predicha por fórmulas ${ }^{15}$.

Por estas razones, establecer valores de referencia y una fórmula para estimar UCr en población chilena era una necesidad no resuelta.

En este trabajo desarrollamos una fórmula que permite predecir los valores de UCr de $24 \mathrm{~h}$ en población chilena a partir de datos demográficos y antropométricos. Concordante a lo descrito en la literatura, la UCr de $24 \mathrm{~h}$ se asoció directamente al peso e inversamente a la edad. El género femenino también se asoció a una menor creatininuria. El determinante más importante de la creatininuria fue el peso, probablemente al ser el factor más asociado a la masa muscular.

El rendimiento de nuestra fórmula es al menos comparable al de las fórmulas publicadas en la literatura. Es destacable que las fórmulas desarrolladas en población predominantemente caucásica (grupo CKD-EPI y Walser) tienen un muy buen rendimiento en nuestra población, lo que no ocurre con las fórmulas desarrolladas en población asiática. Esto reafirma la importancia de que las ecuaciones deben ser validadas en las diferentes poblaciones antes de utilizarse a discreción.

Nuestro trabajo tiene varias fortalezas, entre las que se encuentran entregar rangos de creatininuria para población chilena a partir de un número de datos adecuado, con un amplio rango etario y de función renal. Nuestra fórmula, además, predice los valores de UCr de $24 \mathrm{~h}$ con un excelente rendimiento en comparación a las fórmulas existentes.

La principal limitación de este trabajo es que el desarrollo de la ecuación y su validación se realizaron en poblaciones que, aunque independientes y aleatorias, correspondían al mismo centro de estudios. El tamaño de la población de validación también es limitado. Por lo anterior sería ideal poder probar el rendimiento de esta nueva fórmula en población de otros centros de salud para evaluar su validez externa.

\section{Conclusiones}

En suma, en este trabajo hemos formulado una ecuación para predecir la creatininuria de $24 \mathrm{~h}$ en población chilena. Además, hemos evaluado el rendimiento de las ecuaciones existentes en nuestra población, encontrando que la fórmula propuesta tiene un rendimiento comparable con las fórmulas del grupo CKD-EPI y Walser y superior al del resto de las ecuaciones.

\section{Referencias}

1. Möller E, Mcintosh JF, Van Slyke DD. Studies of urea excretion II: Relationship between urine volume and the rate of urea excretion by normal adults. J Clin Investig. 1928; 6: 427-65.

2. Boyd C, Wood K, Whitaker D, et al. Accuracy in 24hour Urine Collection at a Tertiary Center. Rev Urol. 2018; 20: 119-24. 
3. Mayo Clinic, Mayo Medical Laboratories. Creatinine, 24 hour, urine. https://www.mayomedicallaboratories.com/ test-catalog/Clinical+and+Interpretive/8513 (revisado el 14 de enero de 2020).

4. Snee R. Validation of regression models: methods and examples. Technometrics 1977; 19: 415-28.

5. Xu Y, Goodacre R. On Splitting Training and Validation Set: A Comparative Study of Cross-Validation, Bootstrap and Systematic Sampling for Estimating the Generalization Performance of Supervised Learning. J Anal Test 2018; 2: 249-62.

6. Tanaka T, Okamura T, Miuria K, Kadowaki T, Ueshima $\mathrm{H}$, Nakawawa $\mathrm{H}$, et al. A simple method to estimate populational 24-h urinary sodium and potassium excretion using a casual urine specimen. J Hum Hypertens 2002; 16: 97-103.

7. Ix JH, Wassel CL, Stevens LA, Beck GJ, Froissart M, Navis G, et al. Equations to estimate creatinine excretion rate: the CKD epidemiology collaboration. Clin J Am Soc Nephrol 2011; 6: 184-91.

8. Cockcroft DW, Gault MH. Prediction of creatinine clearance from serum creatinine. Nephron 1976; 16: 31-41.

9. Walser M. Creatinine excretion as a measure of protein nutrition in adults of varying age. J Parenter Enteral Nutr. 1987; 11: 73S-78S.
10. Goldwasser P, Aboul-Magd A, Maru M. Race and creatinine excretion in chronic renal insufficiency. Am J Kidney Di 1997; 30: 16-22.

11. Gerber LM, Mann, SJ. Development of a model to estimate 24-hour urinary creatinine excretion. J Clin Hypertens 2014; 16: 367-71.

12. Rule AD, Bailey KR, Schwartz GL, Khosla S, Lieske JC, Melton III LJ. For estimating creatinine clearance measuring muscle mass gives better results than those based on demographics. Kidney Int 2009; 75: 1071-8.

13. Kawasaki T, Itoh K, Uezono K, Sasaki H. A simple method for estimating $24 \mathrm{~h}$ urinary sodium and potassium excretion from second morning voiding urine specimen in adults. Clin Exp Pharmacol Physiol 1993; 20: 7-14.

14. Jędrusik P, Symonides B, Gaciong Z. Performance of 24-hour urinary creatinine excretion-estimating equations in relation to measured 24-hour urinary creatinine excretion in hospitalized hypertensive patients. Sci Rep. 2019; 9: 3593.

15. Hogan MC, Reich HN, Nelson PJ, Adler SG, Cattran DC, Appel GB, et al. The relatively poor correlation between random and 24-hour urine protein excretion in patients with biopsy-proven glomerular diseases. Kidney Int 2016; 90: 1080-9. 